\title{
Communication \\ High PSRR Wide Supply Range Dual-Voltage Reference Circuit for Bio-Implantable Applications
}

\author{
Ruhaifi Bin Abdullah Zawawi ${ }^{1}$, Hojong Choi ${ }^{2, *}$ and Jungsuk Kim ${ }^{3, *}$ \\ 1 Department of Health Science and Technology, GAIHST, Incheon 21999, Korea; ruhaifi@bme.gachon.ac.kr \\ 2 Department of Medial IT Convergence Engineering, Kumoh National Institute of Technology, 350-27 \\ Gum-daero, Gumi-si 39253, Korea \\ 3 Department of Biomedical Engineering, Gachon University, Hambakmoe-ro 191, Incheon 21936, Korea \\ * Correspondence: hojongch@kumoh.ac.kr (H.C.); jungsuk@gachon.ac.kr (J.K.)
}

Citation: Zawawi, R.B.A.; Choi, H.; Kim, J. High PSRR Wide Supply Range Dual-Voltage Reference Circuit for Bio-Implantable Applications. Electronics 2021, 10, 2024. https:// doi.org/10.3390/electronics10162024

Academic Editors: S.

Abdollah Mirbozorgi and

Nima TaheriNejad

Received: 31 July 2021

Accepted: 19 August 2021

Published: 21 August 2021

Publisher's Note: MDPI stays neutral with regard to jurisdictional claims in published maps and institutional affiliations.

Copyright: (c) 2021 by the authors. Licensee MDPI, Basel, Switzerland This article is an open access article distributed under the terms and conditions of the Creative Commons Attribution (CC BY) license (https:// creativecommons.org/licenses/by/ $4.0 /)$.

\begin{abstract}
On-chip systems are challenging owing to the limited size of the components, such as the capacitor bank in the rectifier. With a small on-chip capacitor, the output voltage of the rectifier might ring if the circuit experiences significant changes in current. The reference circuit is the first block after the rectifier, and the entire system relies on its robustness. A fully integrated dual-voltage reference circuit for bio-implantable applications is presented. The proposed circuit utilizes nonlinear current compensation techniques that significantly decrease supply variations and reject high-supply ripples for various frequencies. The reference circuit was verified using a $0.35 \mu \mathrm{m}$ complementary metal-oxide semiconductor (CMOS) process. Maximum PSRR values of $-112 \mathrm{~dB}$ and $-128 \mathrm{~dB}$ were obtained. With a supply range from 2.8 to $12 \mathrm{~V}$, the proposed design achieves 0.916 and $1.5 \mathrm{mV} / \mathrm{V}$ line regulation for the positive and negative reference circuits, respectively.
\end{abstract}

Keywords: dual-voltage reference circuit; bio-implantable; wide supply range; high PSRR

\section{Introduction}

A block diagram of the bio-implantable system is shown in Figure 1. The external system comprises a coil driver, which wirelessly transmits power to the implanted system. The transmitted power enables the operation of an implantable system comprising a rectifier, reference circuit, low dropout regulator (LDO), digital controllers, analog circuits, and stimulators. The reference circuit is the first block after the rectifier, and the entire system relies on the robustness of the reference circuit. The DC voltage is generated by passing through a rectifier and LDO from an AC power signal, which is wirelessly transmitted via an inductive link [1,2] or infrared radiation [3]. However, the misalignment of the inductively coupled coils or the infrared source sometimes reduces the power transmission efficiency. Consequently, the implanted rectifier produces an unstable output voltage ripple, which is then fed to the input terminal of the reference circuit. Therefore, the voltage reference circuit must provide a stable output over the device process, power supply, and temperature variations. Significant works on reference circuit design have been reported in the literature [4-9], where attempts were made to reduce the variations in the voltage owing to temperature variations. In detail, the prior methods were based on compensating the negative temperature coefficient of a diode-connected bipolar junction transistor (BJT) or complementary metal-oxide semiconductor (CMOS) with a positive temperature coefficient thermal voltage.

The temperature compensation, termed the curvature-compensation technique, of a first-order or higher-order nonlinear voltage or current is often limited by the operating supply voltage. Channel length modulation also occurs in the transistor when the supply voltage increases. A cascode current mirror, which enables high output impedance, was commonly used in earlier studies to suppress the channel length modulation effect. However, at high supply voltages, a phenomenon called impact ionization in metal-oxide 
semiconductor (MOS) transistors creates a leakage current to the substrate that causes problems in the cascode current mirror [10].

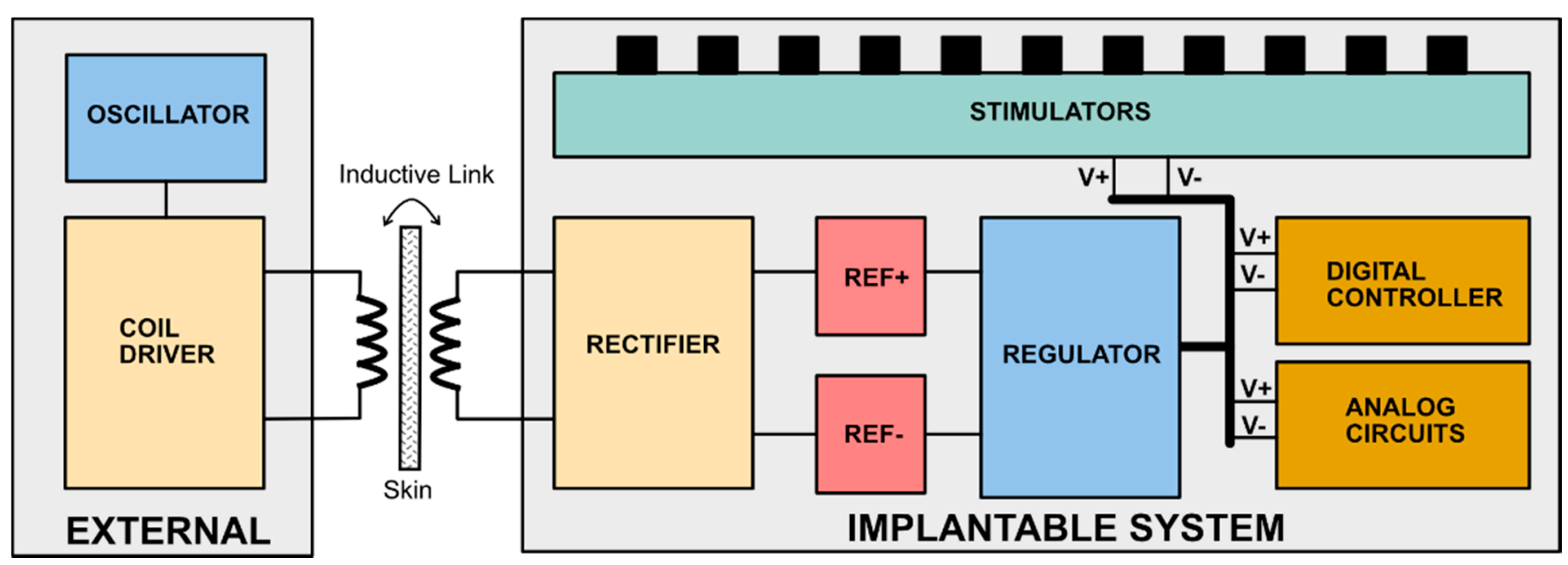

Figure 1. Bio-implantable system diagram.

The voltage across the drain-depletion region and drain current change the magnitude of the substrate current. This is because the drain to the substrate parasitic resistance is created (for the NMOS) in parallel with the output impedance of the MOS transistor. Thus, as the supply increases, the parasitic resistance dominates and significantly reduces the total impedance of the cascode current mirror. This effect produces a significant error in the distributing current to all branches, including the temperature compensation circuits. Consequently, the temperature compensation circuits depend on the supply voltage. Increasing the supply voltage could produce errors in the voltage or current that severely impact the line sensitivity of the reference circuit. Furthermore, these conventional methods require additional circuitry to sink the undesirable current out of the output branch or to add current to the output to compensate for the output voltage variations [11-14], resulting in an expansive layout footprint and higher power consumption. Accordingly, the typical compensation technique for voltage reference is unsuitable for implantable devices. Since our body maintains a constant temperature of $37^{\circ} \mathrm{C}$, it is essential for bio-implantable applications to increase the line regulation efficiency and decrease power dissipation rather than temperature compensation.

Motivated by this, we propose a novel reference circuit optimized for implantable devices that uses nonlinear current compensation circuits to increase the power line regulation performance and power supply rejection ratio (PSRR). The proposed reference circuit was designed using a standard SK-Hynix $0.35 \mu \mathrm{m}$ CMOS standard process.

The current paper extends our previous work in [15]. Our previous work focused on a positive reference only, which was used in LDO to generate a positive supply voltage. However, a negative supply in the stimulators (shown in Figure 1) is also needed to generate balanced biphasic pulses. Therefore, additional circuits, such as a charge pump circuit or inverting voltage circuit, were required in the previous work to generate the negative voltage, increasing the design's total area, due to additional components such as capacitors. The new circuit in this paper fulfils this requirement by having positive and negative references. Furthermore, it utilizes simple compensation circuits to produce excellent performance in PSRR and line regulation for positive and negative reference voltages.

\section{Methods}

The proposed reference circuit diagram is illustrated in Figure 2. The core circuit is based on a self-biasing gate-source voltage reference [6]. The positive reference voltage, $V_{\text {REFP }}$, is the established voltage across the current source circuit when both the current mirror and current source in the core circuit reach the desired operating point, as shown in 
Figure 2. However, an undesired operating point should be avoided because $I_{1}=I_{2}=0$, a start-up circuit guarantees that the proposed circuit is not trapped in the zero-current state by initiating a current in the core circuit. The summation of $V_{G S 1}$ and $V_{G S 2}$ from $V_{G S}=V_{D S, S A T}+V_{T H}$ produces $V_{R E F P}$. The negative reference voltage, $V_{R E F N}$, indicates the voltage generated when $I_{R}$ flows into the resistor, $R$. The three compensation circuits devised in this work are designated as Comp-A, Comp-B, and Comp-C in Figure 2.

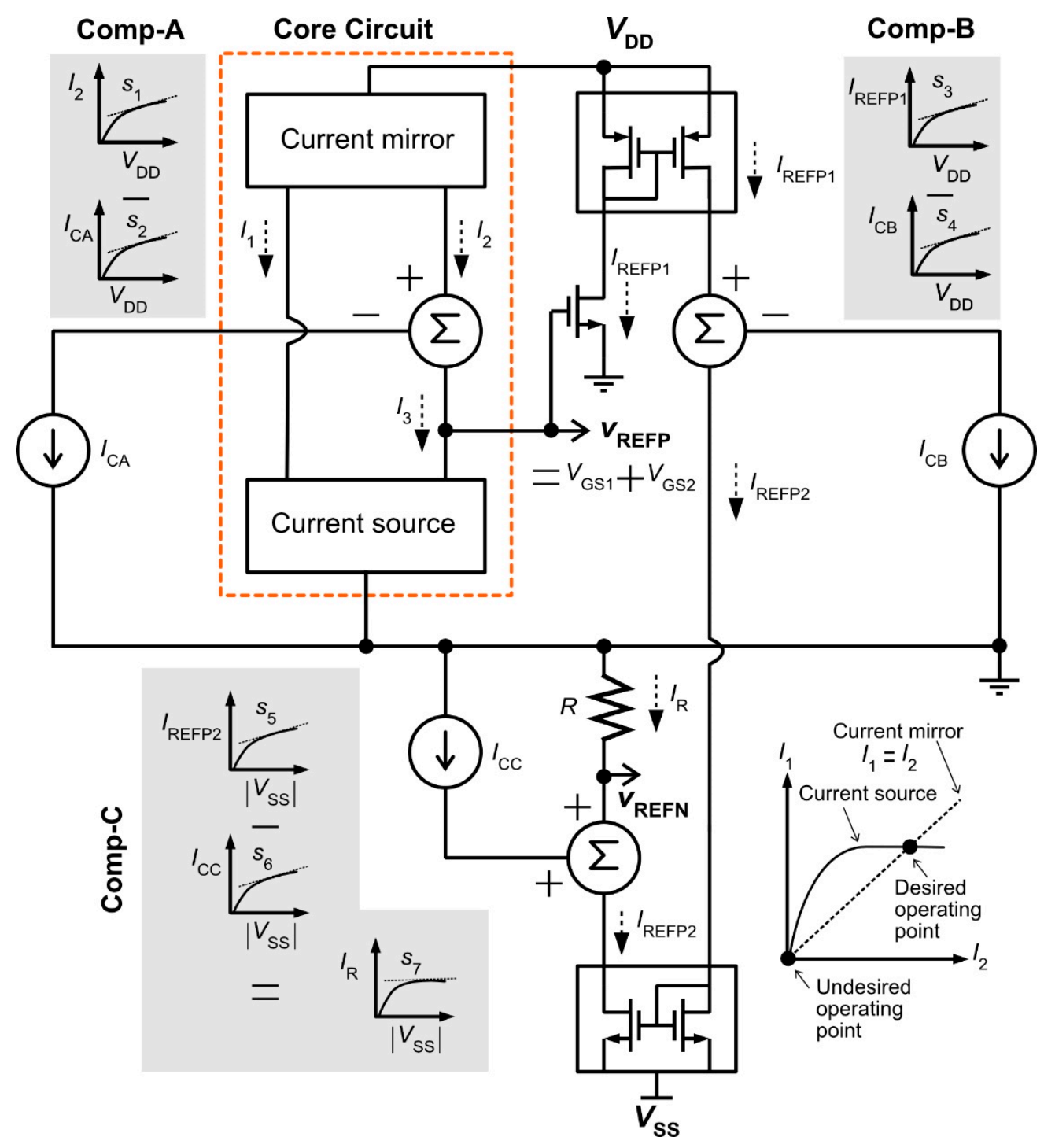

Figure 2. The proposed reference circuit diagram.

Comp-A and Comp-B aim at alleviating the supply-dependent current in $I_{2}$ and $I_{R E F P 1}$, leaving a constant current in the core and current mirror circuits. A constant current can be achieved by equalizing the slopes of $S_{1}$ and $S_{2}$, as well as those of $S_{3}$ and $S_{4}$, respectively, for Comp-A and Comp-B. Comp-C generates the current $I_{C C}$, which is added to the current mirror circuit. Assuming that $I_{C C}$ and $I_{R E F P 2}$ are linear currents, they may be written as

$$
\begin{gathered}
I_{C C}=s_{6} V_{S S}+I_{C C \_D C} \\
I_{\text {REFP2 }}=s_{5} V_{S S}+I_{\text {REFP2_DC }} .
\end{gathered}
$$


where $I_{C C \_D C}$ and $I_{R E F P 2 \_D C}$ are the DC currents of $I_{C C}$ and $I_{R E F P 2}$, respectively. If $S_{5}=S_{6}$, $I_{C C}$ in Equation (1) can be written as

$$
I_{C C}=s_{5} V_{S S}+I_{C C \_D C} .
$$

The current through resistor the $R$ is given by

$$
I_{R}=-I_{C C}+I_{R E F P 2}
$$

Substituting Equations (2) and (3) into Equation (4) gives:

$$
I_{R}=-I_{C C \_} D C+I_{R E F P 2} D C
$$

Equation (5) suggests that an independent supply current $I_{R}\left(S_{7}=0\right)$ is obtained if $S_{5}=S_{6}$ (the detailed equations are provided in Appendix A). The PSRR performance is also increased by incorporating Comp-A, Comp-B, and Comp-C into the proposed circuit. The small-signal analysis that proves this idea will be presented later.

Figure 3 illustrates the proposed dual-voltage reference circuit based on the circuit diagram shown in Figure 2. When the supply is powered up, the start-up circuit sinks some current through $M_{N 2}$, denoted as $I_{S A}$, which then initiates a voltage at the gate terminals of $M_{P 3}$ and $M_{P 4}$. The current starts flowing through $M_{P 3}$ and $M_{P 4}$, thereby establishing $V_{R E F P}$. When $V_{R E F P}$ exceeds the threshold voltage of $M_{N 1}$, current flows through $M_{P 1}$ and $M_{P 2}$. The decrease in voltage at the drain terminal of $M_{N 1}$ moves $M_{N 1}$ into a deep triode region and eventually leads $M_{N 2}$ to operate in a cut-off region. $M_{P 6}, M_{P 5}, M_{P 12}, M_{N 7}$, and $M_{P 13}$ are newly added to the proposed reference circuit that consists of the Comp-A, Comp-B, and Comp-C circuits presented in Figure 2. The slopes of $S_{2}, S_{4}$, and $S_{6}$ shown in Figure 2 can be adjusted by varying the size of transistors $M_{P 6}, M_{N 7}$, and $M_{P 13}$, respectively, as shown in Figure 3. $V_{\text {REFP }}$ can be obtained from the circuit as:

$$
V_{R E F P}=V_{G S, N 3}+V_{G S, N 4}
$$

$M_{N 6}, M_{P 7}$, and $M_{P 9}$ form a current reference circuit, providing $I_{R E F P 1}$ to $M_{P 8}$. The nonlinear current in $I_{R E F P 1}$ is compensated by Comp-B, resulting in $I_{R E F P 2}$, which is mirrored by $M_{N 10}$. The constant current $I_{R}$ produces $V_{R E F N}$ across $R_{2}$, given as:

$$
V_{R E F N}=-I_{R} \times R_{2} .
$$

Since $V_{R E F N}$ becomes more negative if $I_{R}$ increases, the source-gate voltage of $M_{P 13}$ increases and more current flows into $M_{P 13}$. According to Equation (5), the increment in $I_{C C}$ decreases $I_{R}$. Thus, the negative feedback loop returns $V_{R E F N}$ back to its initial value.

Figure 4a shows the equivalent circuit for the positive reference $\left(V_{R E F P}\right)$ small-signal analysis. $v_{R 1}$ can be approximately equal to $v_{\text {refp }}$ because it is the output of the source follower circuit formed by $M_{N 3}$ and $R_{1}$ in Figure 3. Therefore, $v_{g, P 2}$ can be obtained by letting

$$
\left(v_{d d}-v_{g, P 2}\right) g_{m, P 3}=\frac{v_{g, P 2}}{r_{o, N 3}+R_{1}} .
$$

After rearranging Equation (8) for $v_{g, P 2}$, we obtain $v_{g, P 2} \approx v_{d d}$, resulting in $g_{m, P 4}\left(v_{d d}-v_{g, P 2}\right) \approx 0$. Applying nodal analysis at the node of $v_{m}$, the following equation can be derived:

$$
\frac{v_{d d}-v_{m}}{r_{o . P 4}}=g_{m, P 6}\left(v_{m}-v_{r e f p}\right)+\frac{v_{m}-v_{n}}{r_{o, P 6}}+g_{m, N 4}\left(v_{R 1}\right)+\frac{v_{r e f p}}{r_{o, N 4}} .
$$

Assuming that $r_{o, P 6}$ and $r_{o, N 4}$ are large, Equation (9) can be simplified as

$$
\frac{v_{d d}-v_{m}}{r_{o, P 4}} \approx g_{m, P 6}\left(v_{m}-v_{r e f p}\right)+g_{m, N 4}\left(v_{R 1}\right) .
$$


Here, $v_{m}$ can be derived from the circuit written as

$$
v_{m} \approx v_{r e f p}\left(\frac{g_{m, N 4}}{g_{m, P 5}}+1\right)
$$

Substituting Equation (11) into Equation (10), $v_{r e f p} / v_{d d}$ can be obtained as follows:

$$
\frac{v_{r e f p}}{v_{d d}} \approx \frac{1}{r_{o, P 4}}\left(\frac{g_{m, P 5}}{g_{m, P 6} \cdot g_{m, N 4}+g_{m, N 4} \cdot g_{m, P 5}}\right) .
$$

Mathematical analysis shows that $v_{\text {refp }}$ exhibits small variations with $v_{d d} . r_{o, P 4}$ in Equation (12) ensures that the ratio of $v_{r e f p} / v_{d d}$ is nearly zero. The proposed Comp-A contributes $g_{m, P 6}$ in Equation (12), and hence increases the PSRR performance.

A small-signal analysis of the negative reference is illustrated in Figure $4 \mathrm{~b}$. The signal path through $M_{P 10}$ and $M_{P 8}$ is ignored because $I_{R E F P 1}$ is assumed to be an ideal current source. Assuming $g_{m, P 13}, g_{m, N 8} \gg \frac{1}{r_{o, P 13}}, \frac{1}{r_{o, N 8}}$, respectively, the $r_{o}$ of $M_{P 13}$ and $M_{N 8}$ can be neglected. From Figure $4 \mathrm{~b}$, the total resistance, $R_{T O T}$, is extremely high, owing to $r_{0, N 7}$; hence, we can assume that $v_{x} \approx v_{y} \approx-v_{s s}$, resulting in $g_{m, N 10}\left(v_{y}+v_{s s}\right) \approx 0$. Applying nodal analysis at the node of $v_{Z}$, the following equation is obtained:

$$
g_{m, P 13} v_{r e f n}+g_{m, N 8}\left(v_{s s}-v_{z}\right)=\frac{v_{z}+v_{s s}}{r_{o, N 10}} .
$$

and

$$
v_{z}=\frac{v_{r e f n}}{g_{m, N 8} R_{2}}-v_{s s}
$$

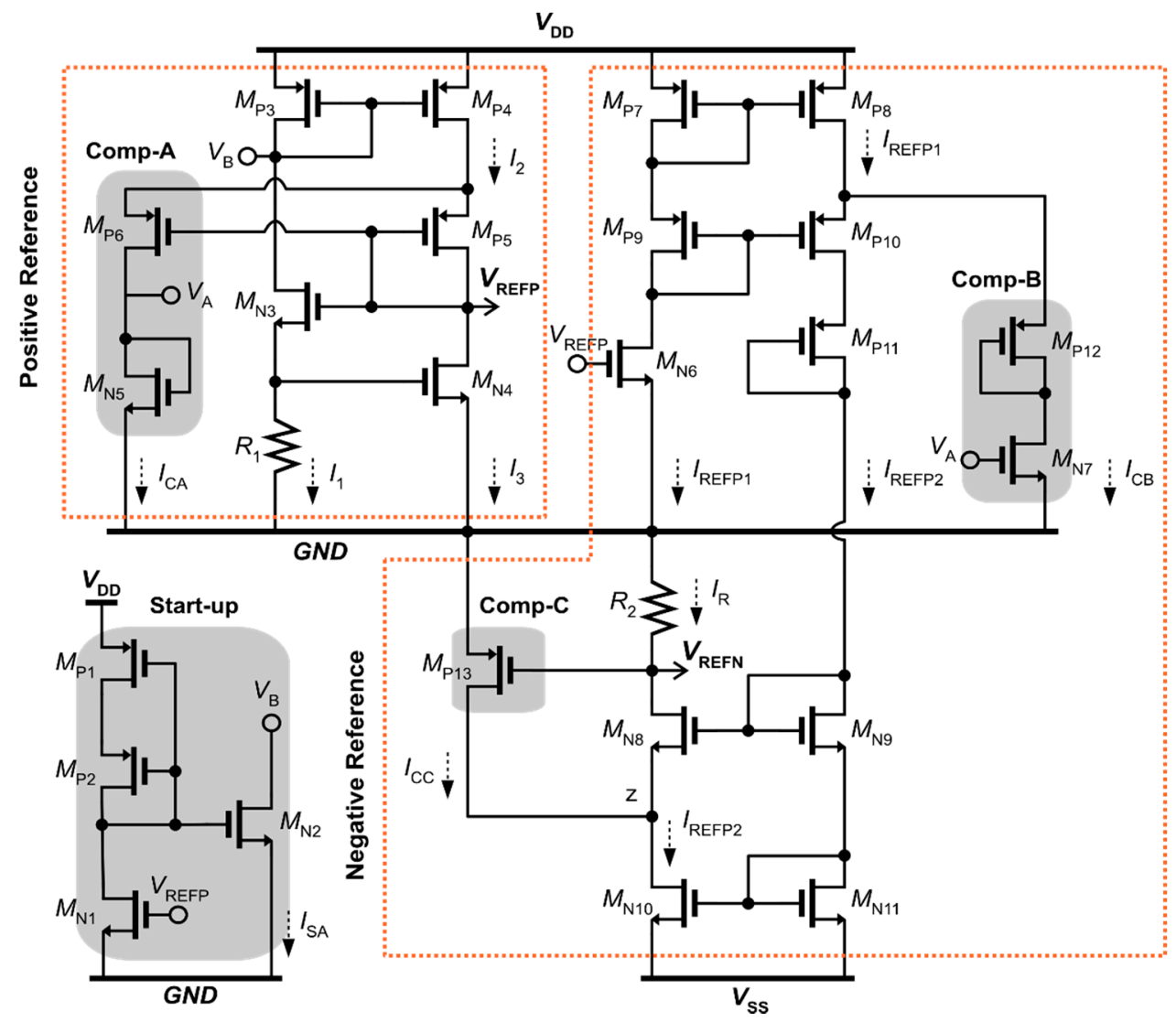

Figure 3. The circuit diagram of the proposed dual-voltage reference circuit. 


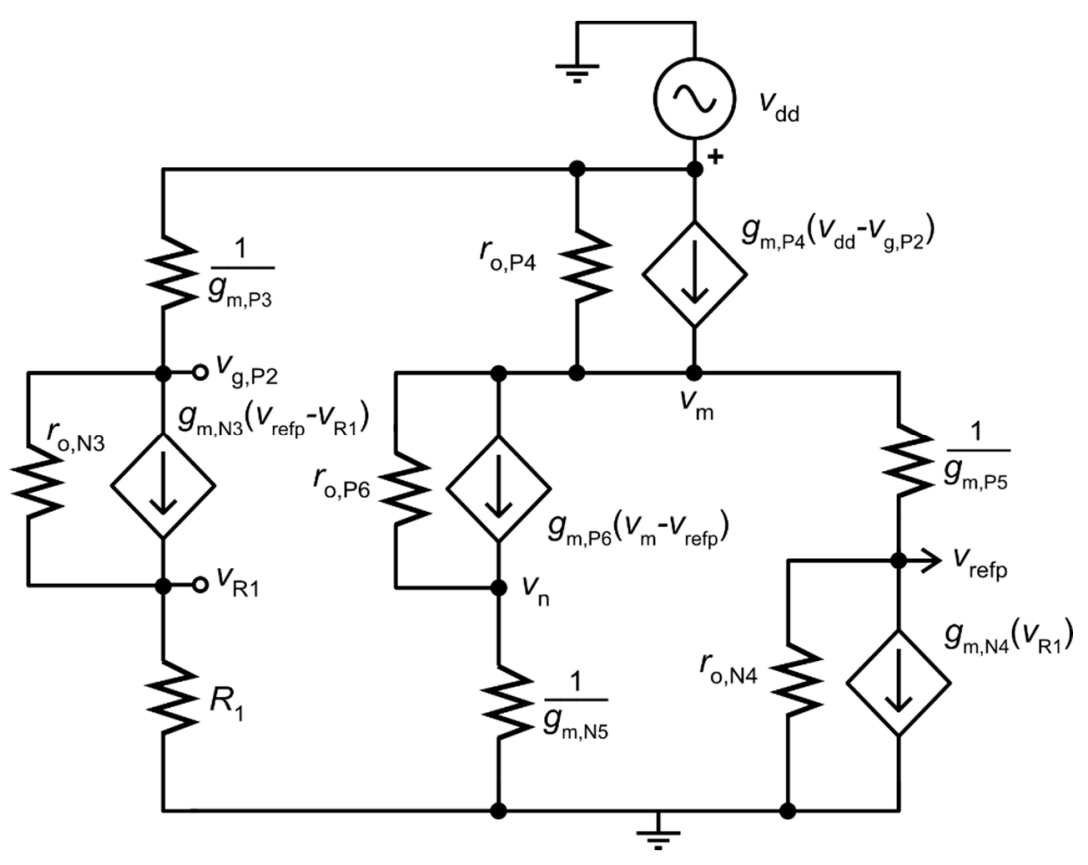

(a)

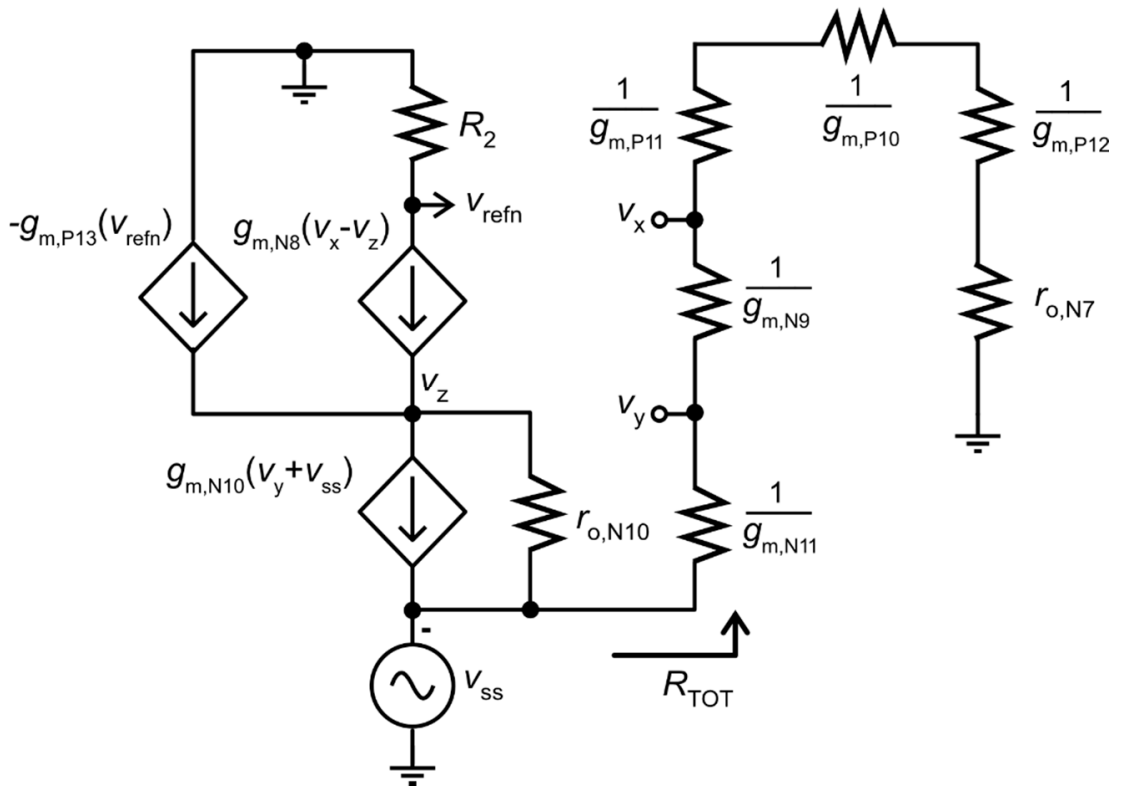

(b)

Figure 4. Small-signal equivalent circuits of the proposed circuit. (a) Positive reference circuit and (b) negative reference circuit.

Substituting Equation (14) into Equation (13) and rearranging for $v_{\text {refn }} / v_{s s}$, we can obtain Equation (15).

$$
\frac{v_{r e f n}}{v_{s S}} \approx \frac{g_{m, N 8} R_{2}}{g_{m, P 13} r_{o, N 10} g_{m, N 8} R_{2}+g_{m, N 8} r_{o, N 10}+1} \approx \frac{R_{2}}{r_{o, N 10}\left(g_{m, P 13} R_{2}+1\right)} .
$$

Equation (15) indicates that $v_{r e f n} / v_{s S}$ is inversely proportional to $r_{o, N 10}$. In addition, $r_{o, N 10}$ is increased by a factor of $g_{m, p 13} R_{2}$, introduced by Comp-C, which decreases the ratio $v_{r e f n} / v_{s s} \cdot g_{m, p 13}$ can be further increased by increasing $I_{C C} ;$ however, $I_{R}$ will be reduced 
slightly, causing $V_{R E F N}$ to become more positive. Hence, the PSRR is limited by the minimum value of $V_{R E F N}$.

The positive and negative reference voltages are fed into the LDO. Thus, PSRR in the LDO is influenced by the reference circuit. The PSRR performance of the LDO also includes an open-loop gain of the error amplifier and output capacitor. In practice, the reference voltage rejects supply noise at a low-frequency region only; hence a high PSRR reference at low frequency is required. The error amplifier maintains a high PSRR in the middle region until it reaches a 3-dB roll-off frequency. The output capacitor of the LDO determines the high-frequency PSRR performance. Table 1 tabulates the component parameters used for the proposed dual-voltage reference circuit.

Table 1. Parameters of the transistors and resistors used for the proposed reference circuit.

\begin{tabular}{cccc}
\hline Component & Parameters & Component & Parameters \\
\hline$M_{P 1}, M_{P 2}$ & $\mathrm{~W}=0.5 \mu \mathrm{m}, \mathrm{L}=25 \mu \mathrm{m}$ & $M_{N 1}$ & $\mathrm{~W}=80 \mu \mathrm{m}, \mathrm{L}=1 \mu \mathrm{m}$ \\
\hline$M_{P 3}, M_{P 4}$ & $\mathrm{~W}=4 \mu \mathrm{m}, \mathrm{L}=1 \mu \mathrm{m}, \mathrm{m}=50$ & $M_{N 2}$ & $\mathrm{~W}=10 \mu \mathrm{m}, \mathrm{L}=1 \mu \mathrm{m}$ \\
\hline$M_{P 5}$ & $\mathrm{~W}=1.15 \mu \mathrm{m}, \mathrm{L}=1 \mu \mathrm{m}$ & $M_{N 3}$ & $\mathrm{~W}=4 \mu \mathrm{m}, \mathrm{L}=1 \mu \mathrm{m}, \mathrm{m}=10$ \\
\hline$M_{P 6}$ & $\mathrm{~W}=4 \mu \mathrm{m}, \mathrm{L}=1 \mu \mathrm{m}, \mathrm{m}=9$ & $M_{N 4}$ & $\mathrm{~W}=100 \mu \mathrm{m}, \mathrm{L}=3 \mu \mathrm{m}$ \\
\hline$M_{P 7}, M_{P 8}$ & $\mathrm{~W}=4 \mu \mathrm{m}, \mathrm{L}=1 \mu \mathrm{m}, \mathrm{m}=4$ & $M_{N 5}$ & $\mathrm{~W}=4 \mu \mathrm{m}, \mathrm{L}=1 \mu \mathrm{m}, \mathrm{m}=20$ \\
\hline$M_{P 9}, M_{P 10}$ & $\mathrm{~W}=16 \mu \mathrm{m}, \mathrm{L}=1 \mu \mathrm{m}$ & $M_{N 6}$ & $\mathrm{~W}=1 \mu \mathrm{m}, \mathrm{L}=10 \mu \mathrm{m}$ \\
\hline$M_{P 11}$ & $\mathrm{~W}=4 \mu \mathrm{m}, \mathrm{L}=1 \mu \mathrm{m}$ & $M_{N 7}$ & $\mathrm{~W}=4 \mu \mathrm{m}, \mathrm{L}=2 \mu \mathrm{m}, \mathrm{m}=3$ \\
\hline$M_{P 12}$ & $\mathrm{~W}=1 \mu \mathrm{m}, \mathrm{L}=50 \mu \mathrm{m}$ & $M_{N 8}, M_{N 9}$ & $\mathrm{~W}=5 \mu \mathrm{m}, \mathrm{L}=10 \mu \mathrm{m}$ \\
\hline$M_{P 13}$ & $\mathrm{~W}=4 \mu \mathrm{m}, \mathrm{L}=1 \mu \mathrm{m}, \mathrm{m}=62$ & $M_{N 10}, M_{N 11}$ & $\mathrm{~W}=1 \mu \mathrm{m}, \mathrm{L}=10 \mu \mathrm{m}$ \\
\hline$R_{1}$ & $70 \mathrm{k} \Omega$ & $R_{2}$ & $150 \mathrm{k} \Omega$ \\
\hline
\end{tabular}

\section{Results}

The proposed reference circuit was verified using a Hynix $0.35 \mu \mathrm{m}$ CMOS standard process. As shown in Figure 5a, $V_{G S, N 4}$ decreases, whereas $V_{G S, N 3}$ increases with increasing $V_{D D}$. As $I_{C A}$ in Comp-A increases rapidly at higher $V_{D D}, V_{R E F P}$ decreases. Thus, the slope of $V_{G S, N 3}$ is assigned to be more positive than $3.5 \mathrm{mV} / \mathrm{V}$ to compensate for the variations at higher $V_{D D} . M_{N 7}$ in Comp-B senses the variations in $V_{D D}$ and produces $I_{C B}$, as shown in Figure 5a, which compensates for the current variation in $I_{R E F P 1}$. The slope of $I_{C B}\left(S_{4}\right.$ in Figure 2) can be adjusted by varying the size of $M_{N 7}$. Comp-C tracks the changes in voltage at node $z$, as shown in Figure 3 , and produces $I_{C C}$, which varies in the same manner as $I_{R E F P 2}$, owing to channel-length modulation. $I_{C C}$ and $I_{R E F P 2}$ vary from $10 \mu \mathrm{A}$ to $19 \mu \mathrm{A}$ and $15.7 \mu \mathrm{A}$ to $24.7 \mu \mathrm{A}$, respectively, and consequently, a stable $I_{R}$ of $5.7 \mu \mathrm{A}$ is obtained when $V_{S S}$ ranges from $2.8 \mathrm{~V}$ to $12 \mathrm{~V}$.

The variations in output voltage with respect to $V_{D D}$ and $V_{S S}$ are presented in Figure 5b. For bio-implantable applications, that is, retinal prosthetics, it is crucial to generate a negative supply voltage for producing a cathodic waveform in a biphasic pulse [14]. Accordingly, we observed a negative output reference voltage with a supply dependency of $1.583 \mathrm{mV} / \mathrm{V}$. As shown in Figure 5a, the nonlinear $I_{C C}$ increases in a similar fashion to $I_{C A}$; however, $V_{G S, N 3}$ compensates for the $V_{R E F P}$ variation caused by $I_{C A}$. Conversely, $V_{R E F N}$ varies at a high supply voltage owing to unmatched current rate changes in $I_{C C}$ and $I_{R E F P 2}$. Thus, the negative reference line regulation is larger than the positive reference line regulation $(0.916 \mathrm{mV} / \mathrm{V})$.

The elaborate corner results for line regulation are shown in Figure $5 c$, according to which the compensation circuits proposed in this work can generate stable reference voltages in various environments. Furthermore, the positive and negative PSRR results with respect to $V_{D D}$ and $V_{S S}$ are plotted in Figure $5 \mathrm{~d}$, where the maximum PSRRs of -112 and $-128 \mathrm{~dB}$ were obtained for $V_{R E F P}$ and $V_{R E F N}$, respectively. The PSRR difference between $V_{R E F P}$ and $V_{R E F N}$ arises from the different factors in the denominator; $r_{0, P 4}$ in 
Equation (12) increases by a factor of $g_{m, N 4}\left(g_{m, p 5}+g_{m, p 6}\right)$, while $r_{o, N 10}$ in Equation (15) increases by approximately $g_{m, P 13}$.

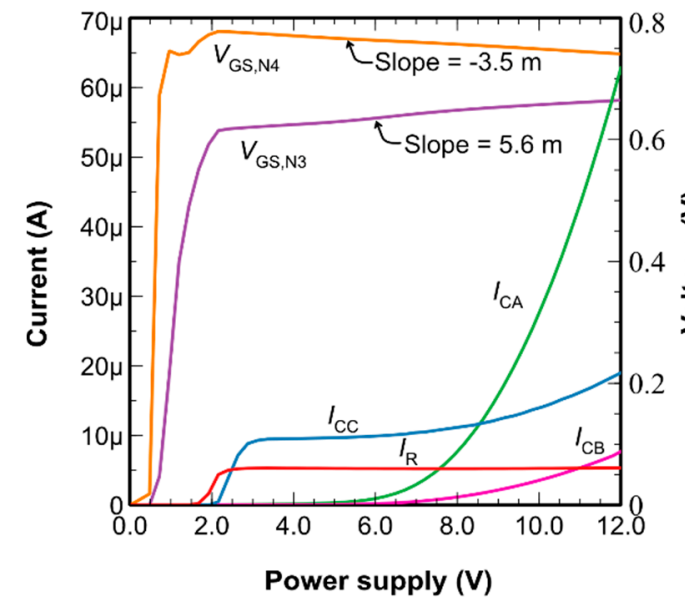

(a)

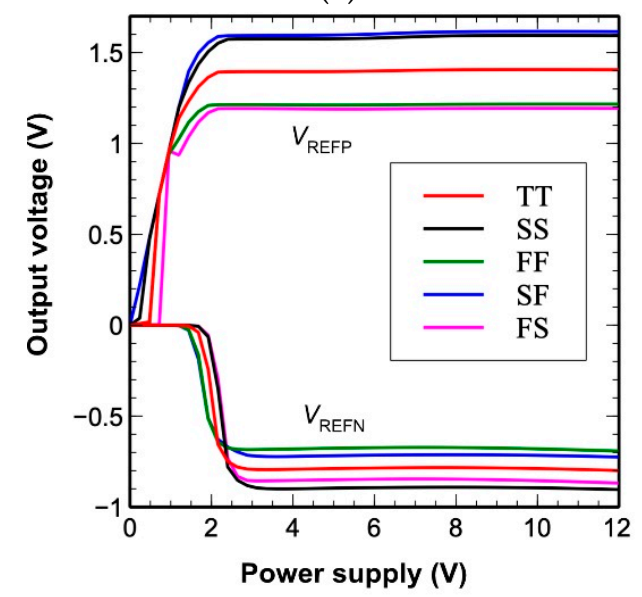

(c)

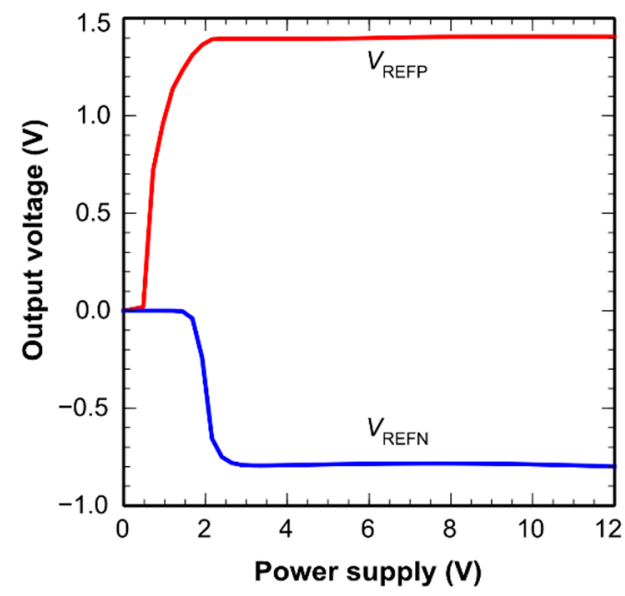

(b)

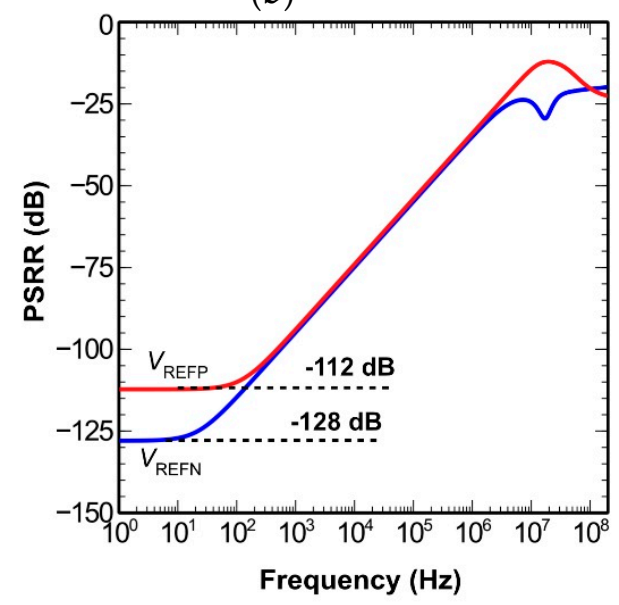

(d)

Figure 5. Results of (a) DC responses of $V_{G S, N 3}, V_{G S, N 4}, I_{C A}, I_{C B}, I_{C C}$, and $I_{R}$, (b) line regulation at typical condition $\left(0 \mathrm{~V} \leq V_{D D} \leq 12 \mathrm{~V}\right.$ and $\left.-12 \mathrm{~V} \leq V_{S S} \leq 0\right)$, (c) line regulation in different corners, and (d) PSRR.

Finally, the overall performance of the proposed reference is tabulated in Table 2 and compared with the previous voltage reference circuits presented in $[12,13,16]$. The line regulation obtained was higher than those in [13,16], but lower than that in [12]. However, the proposed reference circuit exhibits excellent PSRR compared with $[12,13,16]$. The voltage range was $9.2 \mathrm{~V}$, close to [13], while the corresponding quantities for [12,16] were $3 \mathrm{~V}$ and $0.5 \mathrm{~V}$, respectively, indicating that the proposed work is robust to wide supply changes. Considering the accuracy and stability, the proposed reference circuit with excellent line regulation and PSRR is suitable for a wide supply and high precision LDO. In addition, the proposed negative reference could possibly replace the charge pump circuit, which is typically used to generate negative voltage. 
Table 2. Overall performance and comparison.

\begin{tabular}{ccccc}
\hline Parameter & This Work & [12] & [13] & [16] \\
\hline Technology $(\mu \mathrm{m})$ & 0.35 & 0.35 & 1.6 & 0.18 \\
\hline Supply voltage $(\mathrm{V})$ & $2.8 \sim 12$ & $2 \sim 5$ & $2.6 \sim 12$ & $1.3 \sim 1.8$ \\
\hline$V_{\text {REF }}(\mathrm{V})$ & $\begin{array}{c}1.4\left(V_{\text {REFP }}\right) \\
-0.8\left(V_{\text {REFN }}\right)\end{array}$ & 1.14 & 1.6 & 1.17 \\
\hline $\begin{array}{c}\text { Line regulation } \\
(\mathrm{mV} / \mathrm{V})\end{array}$ & $\begin{array}{c}0.916\left(V_{\text {REFP }}\right) \\
1.583\left(V_{\text {REFN }}\right)\end{array}$ & 2 & 0.511 & 0.35 \\
\hline PSRR $(\mathrm{dB})$ & $\begin{array}{c}-112\left(V_{\text {REFP }}\right) \\
-128\left(V_{\text {REFN }}\right)\end{array}$ & -61 & -59.2 & -52 \\
\hline
\end{tabular}

\title{
4. Conclusions
}

A high-PSRR wide-supply range dual-voltage reference circuit was proposed. In practice, the reference circuit needs to reject power supply noise only at low frequencies (usually a few tens of $\mathrm{Hz}$ ), because the LDO error amplifier feedback ensures high PSRR for the remaining frequencies. Hence, the PSRR of -112 and $-128 \mathrm{~dB}$ obtained from the positive and negative references, respectively, minimize the supply noise from the implanted LDO at low frequencies. When the supply ranged from 2.8 to $12 \mathrm{~V}$, line regulation performances of 0.916 and $1.583 \mathrm{mV} / \mathrm{V}$ were obtained for the positive and negative references, respectively. The proposed circuit is suitable for retinal prosthetic applications because balanced biphasic pulses in the stimulators can be generated by supplying stable positive and negative voltages, thereby avoiding damage to body tissues. Presently, a back telemetry digital controller and a dual-voltage LDO that incorporates the proposed reference circuit are under development. These designs will be fully integrated with 256-pixel stimulators and implanted in a pig's eyeball after in vitro testing.

Author Contributions: Conceptualization, R.B.A.Z. and J.K.; methodology, R.B.A.Z., H.C. and J.K.; writing—original draft preparation, R.B.A.Z., H.C. and J.K. Supervision, H.C. and J.K.; funding acquisition, H.C. and J.K. All authors have read and agreed to the published version of the manuscript.

Funding: This work was supported by the Korea Medical Device Development Fund grant funded by the Korea government (the Ministry of Science and ICT, the Ministry of Trade, Industry and Energy, the Ministry of Health \& Welfare, the Ministry of Food and Drug Safety) (Project Number: 202017D01). This work was supported by the National Research Foundation of Korea (NRF) grant funded by the Korea government (MSIT) (No. 2020R1A2C4001606).

Data Availability Statement: The data presented in this study are included in the article.

Acknowledgments: The authors would like to express their sincere appreciation to the IC Design Education Center for chip fabrication.

Conflicts of Interest: The authors declare no conflict of interest.

\author{
Abbreviations \\ The following abbreviations are used in this manuscript: \\ PSRR Power supply rejection ratio \\ LDO Low-voltage drop regulator \\ BJT Bipolar junction transistor \\ CMOS Complementary metal-oxide semiconductor \\ MOS Metal-oxide semiconductor \\ NMOS N-channel metal-oxide semiconductor
}




\section{Appendix A}

From the proposed circuit in Figure 3, we have

$$
I_{C C}=I_{D_{P 13}}\left(1+\lambda_{p} V_{S D_{P 13}}\right) .
$$

We can write the slope of $S_{6}$ in Figure 2 as

$$
\frac{\delta I_{C C}}{\delta_{\left|V_{S S}\right|}}=\frac{\delta I_{C C}}{\delta_{V_{S D \_} P 13}} \cdot \frac{\delta V_{S D \_P 13}}{\delta_{\left|V_{S S}\right|}}=S_{6}
$$

where $\frac{\delta I_{C C}}{\delta_{V_{S D_{-} P 13}}}=I_{D_{-} P 13} \cdot \lambda_{p}$ obtained from Equation (A1). For simplification $\frac{\delta V_{S D_{-} P 13}}{\delta_{\left|V_{S S}\right|}}$ is approximated 1 , hence

$$
S_{6}=I_{D_{-} P 13} \cdot \lambda_{p}
$$

From Figure 3, we know

$$
\begin{gathered}
I_{R E F P 2}=I_{R E F P 1}-I_{C B} \text { and } \\
I_{R E F P 1}=I_{D \_N 6}\left(1+\lambda_{n} V_{D S_{N 6}}\right) .
\end{gathered}
$$

The slope of $S_{3}$ is obtained as follows

$$
\frac{\delta I_{R E F P 1}}{\delta_{V_{D D}}}=\frac{\delta I_{R E F P 1}}{\delta_{V_{D S} N 6}} \cdot \frac{\delta V_{D S \_N 6}}{\delta_{V_{D D}}}=S_{3} .
$$

By assuming $\frac{\delta V_{D S_{-} N 6}}{\delta_{V_{D D}}}=1$, hence

$$
S_{3}=I_{D \_N 6} \cdot \lambda_{n} .
$$

The variation of $I_{R E F P 2}$ with respect to $V_{D D}$ can be written as

$$
\frac{\delta I_{R E F P 2}}{\delta_{V_{D D}}}=S_{3}-S_{4}
$$

or

$$
\frac{\delta I_{R E F P 2}}{\delta_{\left|V_{S S}\right|}}=I_{D_{-} N 6} \cdot \lambda_{n}-S_{4}=S_{5} .
$$

$S_{7}$ equal to 0 if $S_{5}=S_{6}$, therefore

$$
I_{D \_N 6} \cdot \lambda_{n}-S_{4}=I_{D_{-} P 13} \cdot \lambda_{p} .
$$

The slope of $S_{5}$ can be controlled by adjusting $S_{4}$. The following derivation is for obtaining $S_{4}$. First, $I_{C B}$ is assumed in the saturation region,

$$
I_{C B}=\frac{1}{2} K_{n}\left(\frac{W}{L}\right)_{N 7}\left(V_{A}-V_{T H}\right)^{2} .
$$

$S_{4}$ is obtained from the following equation:

$$
\frac{\delta I_{C B}}{\delta_{V_{D D}}}=\frac{\delta I_{C B}}{\delta_{V_{A}}} \cdot \frac{\delta V_{A}}{\delta_{V_{D D}}}=S_{4} .
$$

By assuming $\frac{\delta V_{A}}{\delta_{V_{D D}}}=1$, hence

$$
\frac{\delta I_{C B}}{\delta_{V_{D D}}}=K_{n}\left(\frac{W}{L}\right)_{N 7}\left(V_{A}-V_{T H}\right)=S_{4} .
$$


Equation (A13) suggests that the size of $M_{N 7}$ adjusts the slope of $S_{4}$.

\section{References}

1. Jegadeesan, R.; Nag, S.; Agarwal, K.; Member, S. Enabling Wireless Powering and Telemetry for Peripheral Nerve Implants. IEEE J. Biomed. Health Inform. 2015, 19, 958-970. [CrossRef] [PubMed]

2. Lee, B.; Kiani, M.; Ghovanloo, M. A Triple-Loop Inductive Power Transmission System for Biomedical Applications. IEEE Trans. Biomed. Circuits Syst. 2016, 10, 138-148. [CrossRef] [PubMed]

3. Kuo, P.-H.; Wong, O.-Y.; Tzeng, C.-K.; Wu, P.-W.; Chiao, C.-C.; Chen, P.-H.; Chen, P.-C.; Tsai, Y.-C.; Chu, F.-L.; Ohta, J.; et al. Improved Charge Pump Design and Ex Vivo Experimental Validation of CMOS 256-Pixel Photovoltaic-Powered Subretinal Prosthetic Chip. IEEE Trans. Biomed. Eng. 2020, 67, 1490-1504. [CrossRef] [PubMed]

4. Duan, Q.; Roh, J.; Member, S. A 1.2-V 4.2-ppm C High-Order Curvature- Compensated CMOS Bandgap Reference. IEEE J. Solid-State Circuits 2014, 791, 1-9.

5. Andreou, C.M.; Koudounas, S.; Georgiou, J. A novel wide-temperature-range, 3.9 ppm $/{ }^{\circ} \mathrm{C}$ CMOS bandgap reference circuit. IEEE J. Solid-State Circuits 2012, 47, 574-581. [CrossRef]

6. Osaki, Y.; Hirose, T.; Kuroki, N.; Numa, M. 1.2-V supply, 100-nW, 1.09-V bandgap and 0.7-V supply, 52.5-nW, 0.55-V subbandgap reference circuits for nanowatt CMOS LSIs. IEEE J. Solid-State Circuits 2013, 48, 1530-1538. [CrossRef]

7. Lee, J.M.; Ji, Y.; Choi, S.; Cho, Y.C.; Jang, S.J.; Choi, J.S.; Kim, B.; Park, H.J.; Sim, J.Y. A 29 nW bandgap reference circuit. IEEE Int. Solid-State Circuits Conf. (ISSCC) Dig. Tech. Pap. 2015, 100-101. [CrossRef]

8. Ji, Y.; Jeon, C.; Son, H.; Kim, B.; Park, H.-J.; Sim, J.-Y. A $9.3 \mathrm{nW}$ all-in-one bandgap voltage and current reference circuit. IEEE Int. Solid-State Circuits Conf. (ISSCC) 2017, 100-101. [CrossRef]

9. Lee, I.; Sylvester, D.; Blaauw, D. A subthreshold voltage reference with scalable output voltage for low-power IoT systems. IEEE J. Solid-State Circuits 2017, 52, 1443-1449. [CrossRef]

10. Gray, P.; Meyer, R. Analysis and Design of Analog Integrated Circuits; John Wiley and Sons: Hoboken, NJ, USA, 2010.

11. Zhu, G.; Yang, Y.; Zhang, Q. A 4.6-ppm $/{ }^{\circ} \mathrm{C}$ High-Order Curvature Compensated Bandgap Reference for BMIC. IEEE Trans. Circuits Syst. II Express Briefs 2019, 66, 1492-1496. [CrossRef]

12. Zhou, Z.-K.; Shi, Y.; Wang, Y.; Li, N.; Xiao, Z.P.; Wang, Y.K.; Liu, X.L.; Wang, Z.; Zhao, B. A Resistorless High-Precision Compensated CMOS Bandgap Voltage Reference. IEEE Trans. Circuits Syst. I Regul. Pap. 2019, 66, 428-437. [CrossRef]

13. Sodagar, A.M.; Najafi, K. A wide-range supply-independent CMOS voltage reference for telemetry-powering applications. In Proceedings of the 9th International Conference on Electronics, Circuits and Systems, Dubrovnik, Croatia, 15-18 September 2002; Volume 1, pp. 401-404. [CrossRef]

14. Kang, H.; Abbasi, W.H.; Kim, S.-W.; Kim, J. Fully Integrated Light-Sensing Stimulator Design for Subretinal Implants. Sensors 2019, 19, 536. [CrossRef] [PubMed]

15. Zawawi, R.B.A.; Choi, H.; Kim, J. High-PSRR Wide-Range Supply-Independent CMOS Voltage Reference for Retinal Prosthetic Systems. Electronics 2020, 9, 2028. [CrossRef]

16. Kim, M.; Cho, S.H. A 0.0082-mm², 192-nW Single BJT Branch Bandgap Reference in 0.18- $\mu \mathrm{m}$ CMOS. IEEE Solid State Circuits Lett. 2020, 3, 426-429. [CrossRef] 